\title{
THE STRUCTURAL PARAMETERS OF THE MILKY WAY
}

\author{
ROLAND BUSER \\ Astronomical Institute, University of Basel, Switzerland \\ AND \\ JIANXIANG RONG \\ Astronomy Department, Nanjing University, China
}

\begin{abstract}
A new homogeneous catalog of photographic RGU star count and three-color data in seven high-galactic latitude fields has been used to determine the structural parameter values from a large number of multicomponent population models of the Galaxy. The data provide strong evidence of a prominent thick disk component coexisting with a canonical thin disk and a low-density spheroidal halo.
\end{abstract}

Following the definition and calibration of a standard-RGU system via synthetic photometry techniques $[1,2]$, the plate archive of the BaselPalomar-Schmidt high-latitude survey [3] is being used to construct a homogeneous RGU-photometry data base for a reliable determination of the larger-scale structural parameters and metallicity distributions of the stellar population components of the Galaxy. The new catalog completed to date [4] comprises star counts and three-color distributions for about $10^{4}$ stars in seven out of more than a dozen fields of the original survey.

We have analysed the data essentially following the methodology developed in [5] and summarized in [2]. For each field, the observed star counts, $N(G, G-R)$, are compared to the expected counts calculated from each of 12,800 multi-component models of the Galactic stellar distributions, whose parameters are allowed to systematically vary within plausible ranges suggested by previous work. For each model, a $\chi^{2}$-estimate of goodness-of-fit to the observed data is computed for each field, whence a global $\chi^{2}$-value measuring each model's simultaneous fitting to the all-survey observations in seven fields is derived, and the globally best model is identified by $\chi_{\min }^{2}$.

A $\chi^{2}$-curve is then determined for each parameter by allowing it to vary within the adopted range while keeping all other parameters fixed at their 
TABLE 1. Optimum parameter values for combined survey of seven fields

\begin{tabular}{llcccc}
\hline Component & Parameter & Symbol & Unit & $\begin{array}{c}\text { Optimized } \\
\text { Value }\end{array}$ \\
\hline Thin disk & & local density & $\mathrm{n}_{0}$ & ${\text { stars } \mathrm{pc}^{-3}}$ & 0.078 \\
& & scale length & $\mathrm{d}_{1}$ & $\mathrm{kpc}$ & 4.01 \\
& \multirow{2}{*}{ old } & scale height dwarfs & $\mathrm{h}_{1}$ & $\mathrm{pc}$ & 290 \\
& & scale height giants & $\mathrm{h}_{2}$ & $\mathrm{pc}$ & 250 \\
\multirow{3}{*}{ Thick disk } & \multirow{2}{*}{ young } & scale height dwarfs & $\mathrm{h}_{3}$ & $\mathrm{pc}$ & 170 \\
& & local density & $\mathrm{n}_{1}$ & $\mathrm{n}_{0}$ & 0.054 \\
& scale length & $\mathrm{d}_{2}$ & $\mathrm{kpc}$ & 4.25 \\
Halo & scale height & $\mathrm{h}_{4}$ & $\mathrm{kpc}$ & 1.15 \\
& & local density & $\mathrm{n}_{2}$ & $\mathrm{n}$ & 0.0005 \\
& effective radius & $\mathrm{R}_{\text {eff }}$ & $\mathrm{kpc}$ & 2.69 \\
& flattening & $\mathrm{f}$ & - & 0.84 \\
\hline
\end{tabular}

values adopted by the globally best model. In order to explore the statistical significance of the parameter values associated with $\chi_{\min }^{2}$, we also examine their frequency distributions as functions of $\chi^{2}$ for all the models satisfying the condition $\chi^{2} \leq \chi_{\max }^{2} \leq \zeta \chi_{\min }^{2}$, where $\zeta \in[1.1,1.5]$. These distributions serve to determine the statistical weights that are applied in calculating the optimized value for each parameter, which is finally derived taking into account the possible bias induced by parameter coupling resulting from the preceding analysis.

Table 1 summarizes the results obtained for the combined survey of seven fields. The present data strongly favor a thick disk component whose local density is about 5-6\% of the local thin-disk density, and which thus appears to be considerably more prominent than originally thought $[6,7]$, but in good agreement with more recent studies [8]. These results now also allow us to derive the lairye-scale metallicity distributions from the observed $(U-G)$ colors [9].

\section{References}

1. Buser, R. 1978, A\&A 62, 411; A\&A 62, 425

2. Buser, R. and Fenkart, R.P. 1990, A\&A 239, 243

3. Becker, W. 1965, Z.Ap. 62, 54

4. Buser, R., Karaali, S., Topaktas, L., Karatas, Y. 1994, A\&AS (in press)

5. Buser, R. and Kaeser, U. 1985, A\&A 145, 1

6. Gilmore, G. 1984, MNRAS 207, 223

7. Fenkart, R.P. 1989, A\&AS 78, 217; 79, 51; 80, 89; 81, 187

8. Majewski, S.R. 1993, ARA\&A 31, 575

9. Buser, R. and Rong, J.X. 1994, this volume 